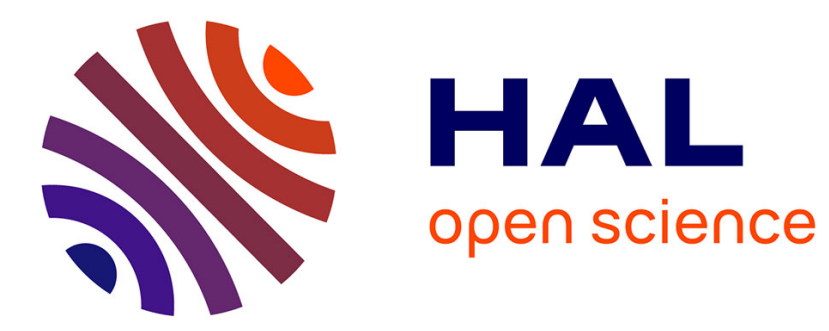

\title{
Model-independent tracking of criticality signals in nuclear multifragmentation data
}

J.D. Frankland, A. Chbihi, A. Mignon, M.L. Begemann-Blaich, R. Bittiger, B. Borderie, R. Bougault, J.-L. Charvet, D. Cussol, R. Dayras, et al.

\section{- To cite this version:}

J.D. Frankland, A. Chbihi, A. Mignon, M.L. Begemann-Blaich, R. Bittiger, et al.. Model-independent tracking of criticality signals in nuclear multifragmentation data. Physical Review C, 2005, 71, pp.034607. 10.1103/PhysRevC.71.034607 . hal-00001474v2

\section{HAL Id: hal-00001474 \\ https://hal.science/hal-00001474v2}

Submitted on 6 Sep 2004

HAL is a multi-disciplinary open access archive for the deposit and dissemination of scientific research documents, whether they are published or not. The documents may come from teaching and research institutions in France or abroad, or from public or private research centers.
L'archive ouverte pluridisciplinaire HAL, est destinée au dépôt et à la diffusion de documents scientifiques de niveau recherche, publiés ou non, émanant des établissements d'enseignement et de recherche français ou étrangers, des laboratoires publics ou privés. 


\title{
Model-independent tracking of criticality signals in nuclear multifragmentation data
}

\author{
J.D. Frankland, ${ }^{1}$ A. Chbihi, ${ }^{1}$ A. Mignon,${ }^{1}$ M.L. Begemann-Blaich, ${ }^{2}$ R. Bittiger,${ }^{2}$ B. Borderie, ${ }^{3}$ R. Bougault,${ }^{4}$ \\ J.-L. Charvet ${ }^{5}$ D. Cussol, ${ }^{4}$ R. Dayras, ${ }^{5}$ D. Durand, ${ }^{4}$ C. Escano-Rodriguez, ${ }^{1}$ E. Galichet,${ }^{3,6}$ D. Guinet,${ }^{7}$ \\ P. Lautesse, ${ }^{7}$ A. Le Fèvre, ${ }^{2}$ R. Legrain, ${ }^{5}$ 用 N. Le Neindre, ${ }^{3}$ O. Lopez, ${ }^{4}$ J. Eukasik, ${ }^{2,8}{ }^{8}$ U. Lynen, ${ }^{2}$ \\ L. Manduci, ${ }^{4}$ J. Marie, ${ }^{4}$ W.F.J. Müller, ${ }^{2}$ L. Nalpas,${ }^{5}$ H. Orth,${ }^{2}$ M. Pârlog, ${ }^{9}$ M. Pichon, ${ }^{4}$ M. F. Rivet ${ }^{3}$ \\ E. Rosato, ${ }^{10}$ R. Roy, ${ }^{11}$ A. Saija, ${ }^{2}$ C. Schwarz, ${ }^{2}$ C. Sfienti, ${ }^{2}$ B. Tamain, ${ }^{4}$ W. Trautmann, ${ }^{2}$ A. Trzcinski, ${ }^{12}$ \\ K. Turzó, ${ }^{2}$ A. Van Lauwe, ${ }^{4}$ E. Vient,${ }^{4}$ M. Vigilante, ${ }^{10}$ C. Volant, ${ }^{5}$ J.P. Wieleczko, ${ }^{1}$ and B. Zwieglinski ${ }^{12}$
}

(INDRA and ALADIN collaborations)

1 GANIL, CEA et IN2P3-CNRS, B.P. 55027, F-14076 Caen Cedex, France.

${ }^{2}$ Gesellschaft für Schwerionenforschung mbH, D-64291 Darmstadt, Germany.

3 Institut de Physique Nucléaire, IN2P3-CNRS, F-91406 Orsay Cedex, France.

4 LPC, IN2P3-CNRS, ENSICAEN et Université, F-14050 Caen Cedex, France.

${ }^{5}$ DAPNIA/SPhN, CEA/Saclay, F-91191 Gif sur Yvette Cedex, France.

${ }^{6}$ Conservatoire National des Arts et Métiers, F-75141 Paris Cedex 03.

${ }^{7}$ Institut de Physique Nucléaire, IN2P3-CNRS et Université F-69622 Villeurbanne, France. 8 Institute of Nuclear Physics, Pl-31342 Kraków, Poland.

${ }^{9}$ National Institute for Physics and Nuclear Engineering, RO-76900 Bucharest-Măgurele, Romania. 10 Dipartimento di Scienze Fisiche e Sezione INFN, Università di Napoli "Federico II", I-80126 Napoli, Italy.

11 Laboratoire de Physique Nucléaire, Université Laval, Québec, Canada. 12 Soltan Institute for Nuclear Studies, Pl-00681 Warsaw, Poland.

(Dated: Revision : 1.2 Date : 2004/09/0613:39:24)

\begin{abstract}
We look for signals of criticality in multifragment production in heavy-ion collisions using modelindependent universal fluctuations theory. The phenomenon is studied as a function of system size, bombarding energy, and impact parameter in a wide range of INDRA data. For very central collisions $\left(b / b_{\max }<0.1\right)$ we find evidence that the largest fragment in each event, $Z_{\max }$, plays the role of an order parameter, defining two different regimes at low and high incident energy, respectively, according to the scaling properties of its fluctuations. Data for a wide range of system masses and incident energies collapse on to an approximately universal scaling function in each regime for the most central collisions. The forms of the scaling functions for the two regimes are established, and their dependence on the total mass and the bombarding energy is mapped out. Data suggest that these regimes are linked to the disappearance of heavy residues in central collisions.
\end{abstract}

\section{INTRODUCTION}

It has long been hoped that the study of nuclear multifragmentation reactions as observed in intermediate energy heavy-ion collisions [1, 2, 3, 4, 5] can give valuable information on the nuclear matter phase diagram and equation of state [6, 7, 8]. The principal guide-wire for this research has been the search for signs of something analogous to a liquid-gas phase transition in data on intermediate mass fragment (IMF) production, ever since the observation of power laws in fragment massyield distributions [9, 10], reminiscent of the condensation of liquid drops in a critical vapour 11]. This effort was encouraged by the failure of statistical models of hot nuclear decay [12, 13, 14] to explain the observed fragment yields 2, 15 unless they suppose the break up of the system at low densities 16, 17, 18 where the phase transition may occur [6].

Signals of the phase transition in experimental data on multifragmentation may be revealed by anomalously

* deceased large fluctuations of fragment observables [19, 20, 21. The main obstacles to such endeavours insofar as experimental data are concerned are the huge statistical fluctuations inherent to small systems such as atomic nuclei, and the still-open question of the mechanism(s) of fragment production in heavy ion collisions at intermediate energies 222, 23, 24, 25, 26, 27, 28, 29, 30, 31, 32, 33. 34, 35, 36, 37, 38, 39. Indeed in all experimental studies of the question, at the same time as one is searching for evidence of the phase transition of a piece of hot nuclear matter [35], one is (implicitly or not) obliged to elucidate the manner in which such an excited system may be formed in the course of certain reactions [34]. The solidity of any experimental evidence for a link between multifragmentation and the nuclear matter phase diagram will be undermined by any remaining ambiguity about the dominant mechanism of fragment production in the selected sample of experimental events, and it would be preferable to be able to address the question of "phases" in nuclear multifragmentation independently of reaction mechanism.

It is for this reason that the theory of the universal character of order parameter fluctuations in finite systems proposed by Botet and Płoszajczak 40, 41, 42, 43 
provides an attractive opportunity to address the question of phase transitions induced by heavy-ion collisions in the least ambiguous way possible. According to their work, it is possible to obtain pertinent information on the relationship between the formation of clusters in a system and the phase transition(s) of said system without needing to characterise the state of the system under study to the extent of, for example, supposing it to be in thermodynamical equilibrium at the time of cluster formation. One needs only to study the properties of the clusters: specifically, all pertinent information can be obtained from a sufficiently precise measurement of the event-byevent distributions of cluster multiplicity and the size of the largest cluster produced. The attractiveness of such an approach in the domain of nuclear fragmentation reactions is obvious, for the reasons given above: although the mechanism of fragment production remains an open question, for several years now large solid-angle multidetector arrays such as INDRA have provided high-quality data on the multiplicity and the size (charge) of the fragments produced in such reactions.

A first application of the universal fluctuations theory to INDRA data for central collisions of $\mathrm{Xe}+\mathrm{Sn}$ from 25 to $50 \mathrm{~A} \mathrm{MeV}$ was published in 44. In this paper we will present the results of the same analysis applied to a wide range of systems measured with INDRA. As in 44 we observe a signature compatible with the existence of different regimes at different bombarding energies. This behaviour disappears for less central collisions. By applying the analysis to data for colliding systems with total mass number between 73 and 394 we will show the mass dependence of the energy of transition between the two regimes, as well as presenting a systematic study of the universal scaling functions observed in the low- and high-energy regimes.

\section{UNIVERSAL FLUCTUATIONS OF THE ORDER PARAMETER IN FINITE SYSTEMS}

Universal scaling laws of fluctuations (the $\Delta$-scaling laws) have been derived for equilibrium systems, and shown to apply also in certain out-of-equilibrium situations. In a system in which the second-order critical behaviour can be identified, the relation between order parameter, criticality and scaling law of fluctuations has been established and the relation between the scaling function and the critical exponents has been found. Details can be found in 43 .

Experimental observables that may be related to a critical order parameter can be identified through their $\Delta$ scaling behaviour. The $\Delta$-scaling is observed when two or more probability distributions $P_{N}[\mathrm{~m}]$ of the observable $m$ for a system of 'size' $N$ collapse onto a single scaling curve $\Phi\left(z_{(\Delta)}\right)$ independent of system size when plotted in terms of the scaling variables:

$$
<m>^{\Delta} P_{N}[m]=\Phi\left(z_{(\Delta)}\right)=\Phi\left(\frac{m-<m>}{<m>^{\Delta}}\right)
$$

where $<m>$ is the mean value of the distribution $P_{N}[m]$ and $\frac{1}{2} \leq \Delta \leq 1$. $<m>$ plays the role of a scale parameter and can replace $N$ as a measure of the size of the system. A less strong (necessary but not sufficient) condition for $\Delta$-scaling is that the variance of the distribution should scale with its mean value as

$$
\sigma^{2} \sim<m>^{2 \Delta}
$$

so that in a log-log plot of $\sigma^{2}$ versus $<m>^{2}$ data should fall on a straight line of slope $\Delta$.

The scaling law Equation 11 with $\Delta=\frac{1}{2}$ is associated with low temperature ("ordered") systems, or with observables which are not related to an order parameter. Scaling with $\Delta=1$ is seen at high temperature ("disordered" system) and also for critical systems. For $m$ to be an order parameter it must exhibit a corresponding change of $\Delta$-scaling regime when some suitable control parameter (e.g. available energy, temperature, bondbreaking probability, etc.) is varied.

Here it is worth saying a word about the comparison of experimental distributions using Eq.11 and the determination of $\Delta$-scaling regimes for data. As the transformation from experimental observable to the scaling variable $z_{(\Delta)}$ is a linear transformation, the form of the $\Phi\left(z_{(\Delta)}\right)$ distribution is the same as that of $P_{N}[m]$. However, the presence of the exponent $\Delta$ in the scale factor $<m\rangle^{\Delta}$ means that two identical distributions will appear different (their widths will be different) if they are scaled using a value of $\Delta$ which is not the one relating the mean values and variances of the two distributions via Eq.22. For example, consider two Gaussian distributions whose widths and mean values are related via Eq.2 with $\Delta=1$. In this case the use of Eq.11 with, for example, $\Delta=\frac{1}{2}$ would not lead to a universal scaling function: the widths of the two scaled distributions would be different, and, because of the normalisation of $\Phi\left(z_{(\Delta)}\right)$, the height of the distributions would differ also. It should also be noted that the relationship between the mean and the variance of a set of distributions, i.e. the value of $\Delta$ if Eq.2 holds, is quite independent of the form of the distributions: a given value of $\Delta$ does not imply a certain type of distribution, and vice versa.

More detailed information on the state of the system and the fragmentation process may be found in the form of the scaling functions $\Phi\left(z_{(\Delta)}\right)$, Eq. 11. For systems far from a critical point, the central limit theorem tells us that for an observable $m$ being the sum of uncorrelated random variables, one should observe asymptotically a Gaussian distribution for fluctuations of $m$ about its mean value. If on the other hand $m$ is an extremal value such as the largest among a set of uncorrelated random variables, then asymptotically its distribution 
should be that of Gumbel's first asymptote 45]. For critical systems such general results do not exist, as, due to the presence of correlations at all length-scales, the order parameter distribution must depend on the precise details of the interaction in this case. An asymptotic form of the large- $m$ scaling function tail was derived in [42], where close to a critical point the order parameter distribution was expected to fall off like $\exp -m^{\hat{\nu}}$ with $3 \leq \hat{\nu} \leq 6$, meaning that large deviations of the order parameter from its mean value are strongly suppressed.

There are two generic families of fragment production scenarios for which the second-order phase transition has been identified, with two different order parameters. These are

- the fragment multiplicity for fragmentation scenarios such as the fragmentation-inactivation binary (FIB) model 46.

- the size of the largest cluster or fragment in the socalled aggregation scenarios such as percolation or Fisher droplet models 42 .

Therefore the elimination of one of these two easily measurable experimental quantities as not having the order parameter $\Delta$-scaling behaviour described above should give important information on the fragment production process, by allowing to exclude one of the two scenarios.

\section{PRESENTATION OF DATA}

\section{A. Experimental details}

In order to study as exhaustively as possible the question of the existence of an order parameter or other "phase-like" behaviour in heavy-ion collisions, we have profited from the wide range of very high quality data which has been obtained with the INDRA $4 \pi$ array 477, 48, 49 at the GANIL (Caen) and GSI (Darmstadt) accelerator facilities. This charged product detector covers about $90 \%$ of the $4 \pi$ solid angle. The total number of detection cells is 336 arranged according to 17 rings centred on the beam axis. The first ring $\left(2^{\circ}-3^{\circ}\right)$ was composed of 12 fast NE102/NE115 phoswich detectors during the experiments at the GANIL facility. For the GSI experiments these were replaced by 12 telescopes composed of a $300 \mu \mathrm{m}$ thick silicon detector and a $14 \mathrm{~cm}$ thick $\mathrm{CsI}(\mathrm{Tl})$ scintillator. Rings 2 to 9 cover the angular range from $3^{\circ}$ to $45^{\circ}$ and are made of three detector layers: a low pressure gas-ionization chamber $\left(5 \mathrm{~cm}\right.$ of $\mathrm{C}_{3} \mathrm{~F}_{8}$ at 20 to 50 mbar, depending on the experiment and the polar angle), a $300 \mu \mathrm{m}$ thick silicon detector and a 14 to $10 \mathrm{~cm}$ thick $\mathrm{CsI}(\mathrm{Tl})$ scintillator. The remaining 8 rings cover the angular range from $45^{\circ}$ to $176^{\circ}$ and have two detection layers: ionization chamber and 7.6 to $5 \mathrm{~cm}$ thick $\mathrm{CsI}(\mathrm{Tl})$ scintillator. Fragments with $Z$ up to the charge of the projectile are identified with unit resolution in the forward region, when they are stopped in the scintillator detectors. Beyond $45^{\circ}$, the charge resolution is one unit up to $Z=16$, and a few charges for larger $Z$. Over the whole angular range, a very good isotope identification is obtained for $Z=1$ to $Z=3$, except for particles with low energies where ambiguities are unresolved.

The energy calibration of the $\mathrm{CsI}(\mathrm{Tl})$ scintillators was obtained for light charged particles (LCP) by means of the elastic and inelastic scattering of secondary LCP beams (p,d,t, ${ }^{3} \mathrm{He},{ }^{4} \mathrm{He}$ ) produced by the fragmentation of a $95 \mathrm{~A} \mathrm{MeV}{ }^{16} \mathrm{O}$ beam in a thick $\mathrm{C}$ target. These particles were then momentum selected by the "alpha magnetic spectrometer" of GANIL and scattered in a C or Ta target installed in the INDRA reaction chamber. A typical energy resolution was about 4\%. Typical identification thresholds are a few $100 \mathrm{keV}$ for light particles, $0.7 A \mathrm{MeV}$ for $Z=3$, and $1.4 A \mathrm{MeV}$ for $Z=35$. A complete technical description of INDRA, its calibration and its electronics can be found in $47,48,49,50,51,52,53$.

\section{B. Overview of data}
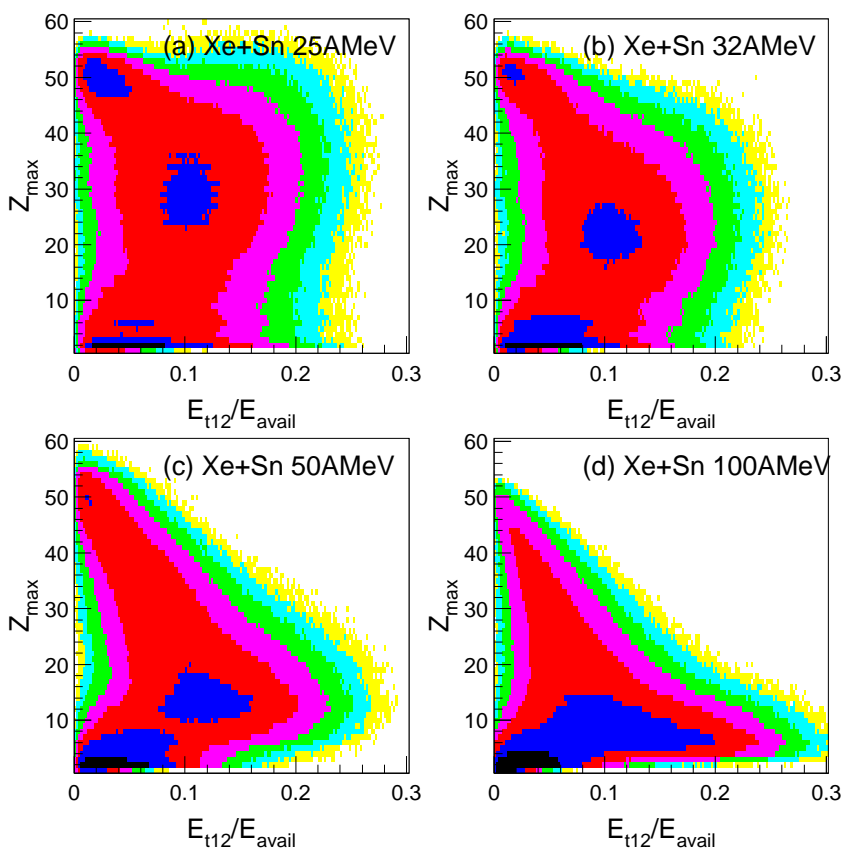

Figure 1: (Color online) Experimental correlations between the size of the largest detected fragment $Z_{\max }$ and the total transverse energy of light charged particles divided by the available c.m. energy, for $\mathrm{Xe}+\mathrm{Sn}$ collisions. A minimum of 4 well-identified charged particles was required in the off-line analysis. Logarithmic contour levels are shown corresponding to the number of events (darker tones indicate larger numbers).

The data presented here cover a wide range of quasisymmetric systems studied with the INDRA array, with different total masses and bombarding energies. They are: 
$\mathrm{Ar}+\mathrm{KCl} \quad 32,40,52$ and $74 \mathrm{~A} \mathrm{MeV;}$

$\mathrm{Ni}+\mathrm{Ni} \quad 32,40,52,63,74,82$ and $90 A \mathrm{MeV}$;

$\mathrm{Xe}+\mathrm{Sn} \quad 25,32,39,45,50,65^{*}, 80^{*}$ and $100^{*} A \mathrm{MeV}$;

$\mathrm{Au}+\mathrm{Au} \quad 40^{*}, 60^{*}$ and $80^{*} A \mathrm{MeV}$.

The systems marked with an asterisk were measured during the experimental campaign at the GSI facility. We have concentrated on symmetric colliding systems in order to benefit from the maximum overall efficiency of the INDRA array in this case. Before presenting the analysis of this data set in terms of universal fluctuations we will give an overview of the chief characteristics of these reactions.

Figure 1 shows experimental data for the $\mathrm{Xe}+\mathrm{Sn}$ system. The contour plots show the number of events measured corresponding to each value of the size of the largest detected fragment, $Z_{\max }$, and of the fraction of the available energy converted into transverse energy of light charged particles, $E_{t 12} / E_{\text {avail }}$. This latter quantity has been shown [29, 31, 54, 55] to be principally related to the geometry of heavy-ion collisions in this energy domain, and is particularly well-suited to sorting events measured with the INDRA detector with little bias, because the efficiency of the array for light charged particle detection is $\approx 90 \%$ whatever the centrality/reaction mechanism. The data shown were recorded with an online trigger requiring that at least four detectors fired in coincidence, while in the off-line analysis we required at least four correctly identified charged products in each considered event. Very similar plots were obtained for all other data studied in this paper, as for example the data for the $\mathrm{Ni}+\mathrm{Ni}$ system shown in Figure 2. They give an overview of the evolution of reaction mechanisms with beam energy and impact parameter.

For the least violent collisions (small $E_{t 12} / E_{\text {avail }}$ ) two strong contributions to the total cross-section are observed, depending on whether the projectile-like fragment was detected $\left(Z_{\max } \approx Z_{\text {proj }}\right)$ or not $\left(Z_{\max }<10\right)$. For most of these collisions the target-like residue is too slowmoving to be detected and/or correctly identified by the INDRA array. With increasing beam energy, the contribution from projectile-like fragments appears to decrease in importance. This is due to the increasingly forwardfocused kinematics of the reactions and the smaller grazing angle at higher energy, which mean that projectilelike fragments from peripheral collisions are more likely to be deflected to angles too small for them to be detected in the first ring of the INDRA array (see III A).

For more central collisions (larger $E_{t 12} / E_{\text {avail }}$ ) the outcome of the reaction depends on bombarding energy and the mass of the colliding nuclei. For the $\mathrm{Xe}+\mathrm{Sn}$ system at the lowest incident energies (Figures 1 $1 \mathrm{a}-\mathrm{b}$ ), the cross-section for all but the most peripheral collisions is dominated by events for which the heaviest detected fragment has around one half of the charge of the projectile nucleus, and there is a discontinuous evolution in cross
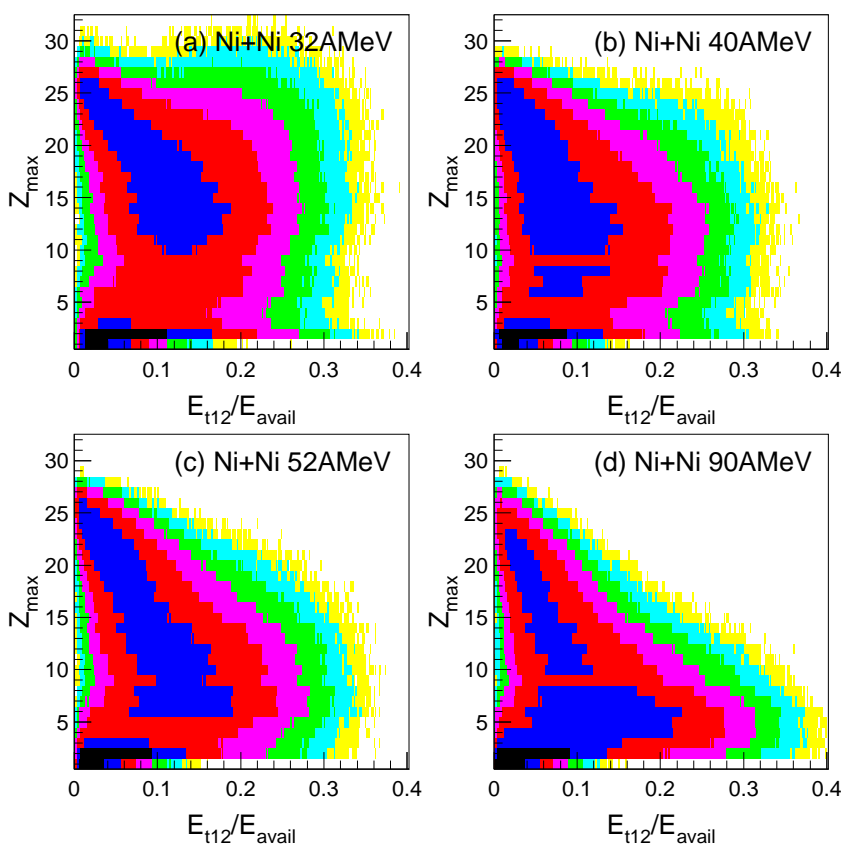

Figure 2: (Color online) Experimental correlations between the size of the largest detected fragment $Z_{\max }$ and the total transverse energy of light charged particles divided by available c.m. energy, for $\mathrm{Ni}+\mathrm{Ni}$ collisions. A minimum of 4 wellidentified charged particles was required in the off-line analysis. Logarithmic contour levels are shown corresponding to the number of events (darker tones indicate larger numbers).

section between the most peripheral collisions and these events. This is due to the onset of fission of projectile-like nuclei above a certain threshold excitation energy which is attained in mid-peripheral and more central reactions 55]. At higher bombarding energies (Figures [1 c-d) a more continuous evolution of projectile-like residue size with the collision violence is seen. For a lighter system such as $\mathrm{Ni}+\mathrm{Ni}$ (Figures 2a-b) a continuous ridge goes from the most peripheral toward more central collisions showing that the size of the projectile-like residues decrease continuously with decreasing impact parameter, as fission is not a predominant decay channel even at high excitation energies for such light nuclei. The correlations between $Z_{\max }$ and $E_{t 12} / E_{\text {avail }}$ for the $\mathrm{Ar}+\mathrm{KCl}$ system (not shown) are very similar to those for $\mathrm{Ni}+\mathrm{Ni}$, whereas for the $\mathrm{Au}+\mathrm{Au}$ system they resemble those for $\mathrm{Xe}+\mathrm{Sn}$ at 50 and $100 \mathrm{~A} \mathrm{MeV}$ except that for the most peripheral collisions a clear contribution from the fission of gold quasiprojectiles is seen.

Finally let us remark the similarity between Figures 17a and 2a, concerning the production of heavy residues at low incident energy. Both figures show that there are two distinct contributions to the total yield for residues with $Z$ close to that of the projectile. The first was discussed in the previous paragraph and is due to projectilelike fragments produced in peripheral collisions (small $E_{t 12} / E_{\text {avail }}$ values). This is the dominant contribution. 
Nevertheless there is a second contribution which is associated with a broad distribution of large residue sizes $Z_{\text {proj }} / 2<Z_{\text {max }} \lesssim Z_{\text {proj }}$ as well as an equally broad distribution of $E_{t 12} / E_{\text {avail }}$ values corresponding to mid- to central collisions. The appearance of these experimental correlations suggests that the mean value of the charge of the heaviest detected fragment, $\left\langle Z_{\max }\right\rangle$, first decreases with increasing collision violence and then increases for the most "central" collisions at the lowest beam energy
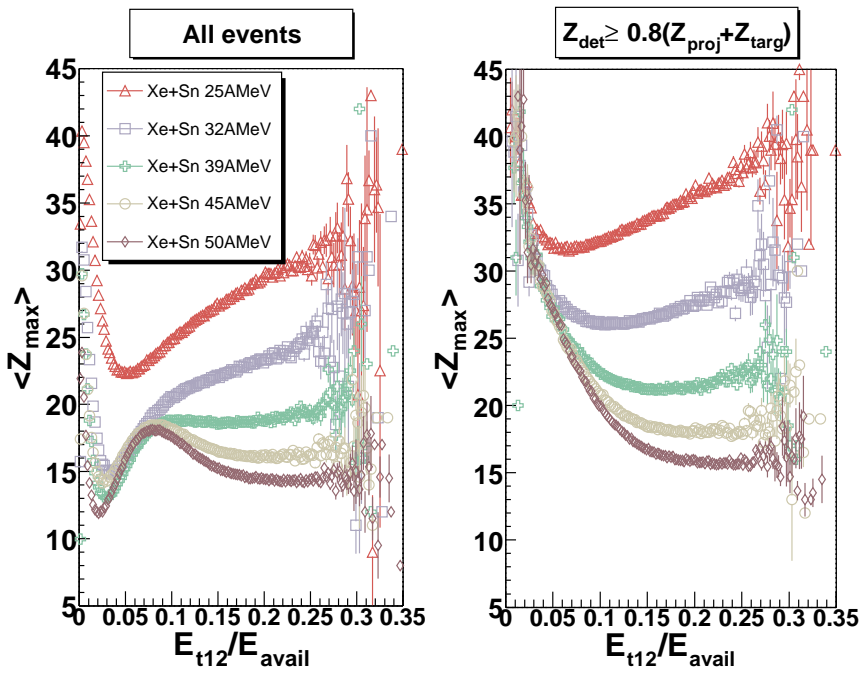

Figure 3: (Color online) The mean charge of the largest fragment detected in each event, $Z_{\max }$, as a function of the total transverse energy of light charged particles (normalised to the available centre of mass energy) for $\mathrm{Xe}+\mathrm{Sn}$ collisions between 25 and $50 \mathrm{~A} \mathrm{MeV}$. Vertical bars show the estimated statistical error on the mean. It should be noted that events with $E_{t 12} / E_{\text {avail }} \gtrsim 0.2$ correspond to only $1 \%$ of the total measured cross-section.

for these two systems.

According to Figures 11 and 2 such reactions make a relatively important contribution to the production of heavy residues in central collisions of $\mathrm{Ni}+\mathrm{Ni}$ and $\mathrm{Xe}+\mathrm{Sn}$ at 32 and $25 \mathrm{~A} \mathrm{MeV}$ respectively. When the incident energy increases ( $40 A \mathrm{MeV}$ for $\mathrm{Ni}+\mathrm{Ni}$, Figure $2 \mathrm{~b}$, and $32 A$ $\mathrm{MeV}$ for $\mathrm{Xe}+\mathrm{Sn}$, Figure $\mathrm{l} \mathrm{b}$ ) the size and yield of the associated residues decrease making this contribution harder to distinguish. From Figures 20 c-d and Figures 1 $\mathrm{k}$-d it appears that the cross-sections associated with these reactions become very small above $\sim 40 \mathrm{AMeV}$, for the $\mathrm{Ni}+\mathrm{Ni}$ system, or above $\sim 32 \mathrm{AMeV}$, for the $\mathrm{Xe}+\mathrm{Sn}$ system (for the $\mathrm{Ar}+\mathrm{KCl}$ system, not shown here, this contribution is discernible up to $52 \mathrm{~A} \mathrm{MeV}$ ). However, more sensitive analyses (e.g. see below for $\mathrm{Xe}+\mathrm{Sn}$ ) can reveal the survival of such reactions at higher incident energies.

Figure 3 shows the evolution of $\left\langle Z_{\max }\right\rangle$ with collision centrality for the $\mathrm{Xe}+\mathrm{Sn}$ system between 25 and $50 \mathrm{~A}$ $\mathrm{MeV}$. It can be seen that the mean charge of the heaviest fragment produced in each event increases with increasing centrality for 25,32 , and, very slightly, $39 A \mathrm{MeV}$. It should be noted that this tendency is observed whether one considers all (Figure 3 left panel) or only wellmeasured (right panel - ratio of total detected charge, $Z_{\text {det }}$, to total charge of projectile and target required to be at least $80 \%$ ) events. The requirement of wellmeasured events reveals the monotonous decrease of the mean charge of projectile-like fragments in peripheral collisions $\left(E_{t 12} / E_{\text {avail }}<0.1\right)$ by excluding from the average those events where neither target-like nor projectile-like fragments were detected (compare Figure 11).

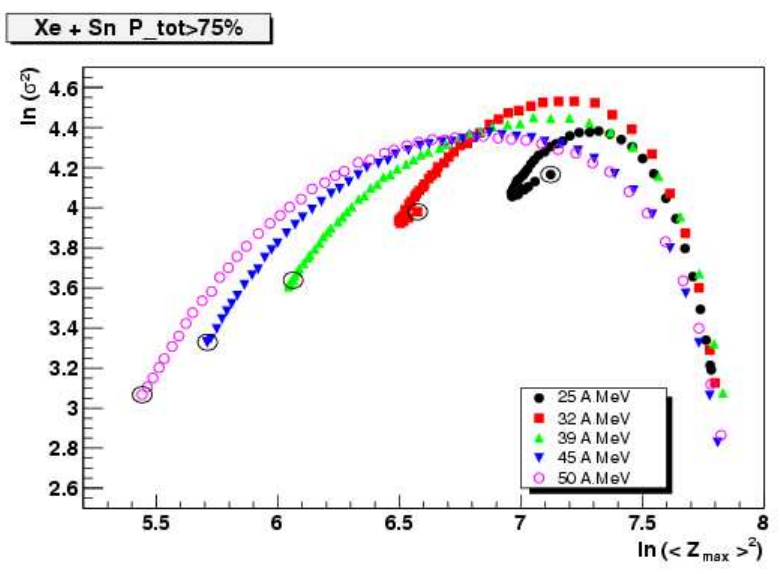

Figure 4: (Color online) Collisions from the most peripheral with detection of a quasi-projectile residue (furthest righthand side of the figure) to the most central (indicated by a ring) are classed into bins corresponding to $1 \%$ of the total measured cross section.

Another way of excluding poorly-measured events is to keep only those for which most of the momentum of the incident beam particles is reconstructed from the detected nuclei. This gate on data has the effect of retaining all events for which at least a good reconstruction of the quasi-projectile residue and products was obtained, for the most peripheral collisions, whilst in the most central collisions we achieve an almost complete reconstruction of the event. In this way we can follow, in Figure 4 , the correlated evolution of the variance and the mean of the $Z_{\max }$ distribution as a function of collision violence: from peripheral collisions leading to a slightly excited quasi-projectile (large $\left\langle Z_{\max }\right\rangle$ and small variance) to the most central collisions (indicated by a ring around the last data point for each beam energy). Each point in this figure corresponds to $E_{t 12}$ cuts defined by slicing the minimum-bias distribution into 100 bins, each containing an equal number of events. Thus each point represents $1 \%$ of the measured cross-section, although due to our requirement of well-measured events the most peripheral (least well measured) events are absent. It allows to see how in the most central collisions two different behaviours are observed depending on the incident beam energy: below $39 \mathrm{~A} \mathrm{MeV}$ in the $3-5 \%$ most central collisions the mean charge of the largest fragment actually begins to increase again, whilst the correlation between this mean value and the variance of the distribution is not 
the same as for the majority of other impact parameters.

This figure suggests that at beam energies $\lesssim 39 A$ $\mathrm{MeV}$ the origin of the heaviest fragment is not the same in central collisions as for the rest of the reactions. The increase of $\left\langle Z_{\max }\right\rangle$ for the most central collisions at these energies suggests a contribution from (incomplete) fusion reactions where the heaviest fragment detected is an evaporation residue. The disappearance of this phenomenon for $\gtrsim 39 \mathrm{~A} \mathrm{MeV}$ can be interpreted as signalling the disappearance of fusion-evaporation residues, and marking the onset either of fusion-multifragmentation [34 or of transparency in central collisions (incomplete stopping) [30, 56, 57].

\section{ANALYSIS OF DATA IN TERMS OF UNIVERSAL FLUCTUATIONS}

\section{A. Selection of central collisions}

We begin our analysis by extending the results of 44, for central collisions of $\mathrm{Xe}+\mathrm{Sn}$, to a wider range of systems measured with the INDRA multidetector array. As was discussed in Sec. If, the analysis of universal fluctuations in multifragmentation data does not require to know (or to suppose) how fragments are formed. However the comparison of events with very different collision geometries is unlikely to give meaningful results. Therefore we will limit our study to very central collisions for which the geometrical overlap between projectile and target is as close as possible to total. The need to select equivalent classes of events for a large range of system masses and beam energies with as little dependence as possible on detector efficiency led us to use the total transverse energy of light charged particles, $E_{t 12}$.

The sorting variable $E_{t 12}$ has been studied specifically for the INDRA detector [31, 58]. It was shown that, for a given projectile-target system, the minimum-bias inclusive distributions of $E_{t 12}$ for different beam energies scale as a function of the available centre of mass energy, consistent with $E_{t 12}$ being mainly sensitive to the geometry of the collisions, i.e. that it is a good indicator of collision centrality. Moreover, as it was pointed out in Sec. III B, the efficiency of the INDRA detector for light charged particles is almost independent of the type of reaction under study, allowing to use this variable to sort all data considered in this paper in the same way.

In addition, in this way we avoid any direct link between the studied observables (size of the largest fragment in each event and fragment multiplicity) and the variable used for the selection of events. We are therefore in the best possible situation to avoid distortion of the data due to autocorrelations with the experimental filter 15 .

The data analysed in the following with the largest values of $E_{t 12}$ correspond to $1 \%$ of the total number of events recorded during the experiment with a minimumbias condition $\left(b<0.1 b_{\max }\right.$ in the geometrical approx- imation of 59 where the maximum impact parameter $b_{\max }$ is smaller than the sum of the radii of projectile and target due to the experimental trigger condition). We were able to check whether the largest detected charged fragment of each event is really the largest, using the total detected charge of the event, $Z_{t o t}$.

\section{B. Establishing an "order parameter" for nuclear multifragmentation with no model-dependent hypotheses}
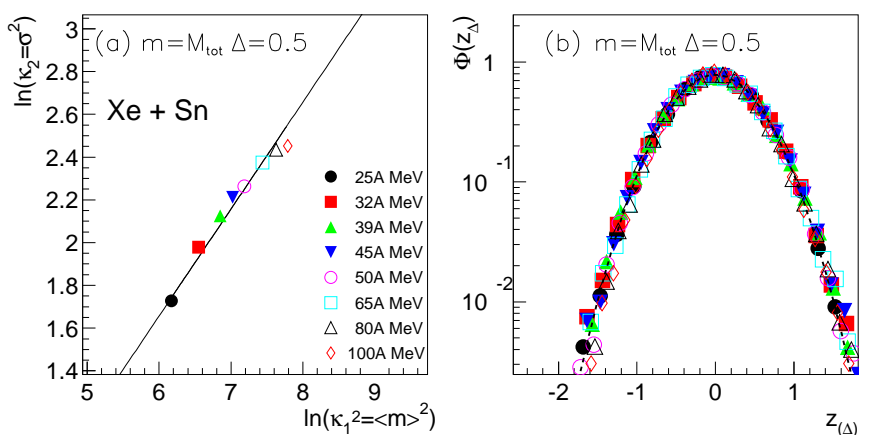

Figure 5: (Color online) Data for the total multiplicity of charged products with $Z \geq 1, M_{t o t}$, for well-measured central collisions of $\mathrm{Xe}+\mathrm{Sn}\left(b<0.1 b_{\text {max }}, Z_{\text {det }} \geq 0.8\left(Z_{\text {proj }}+Z_{\text {targ }}\right)\right)$. (a) $\log -\log$ plot of the variance versus the squared mean value of the distribution of $M_{t o t}$ for each bombarding energy. The straight line represents Eq. 2 with $\Delta=\frac{1}{2}$. (b) Experimental distributions of $M_{t o t}$ expressed in the variables of the second scaling law, i.e. Equation f with $\Delta=\frac{1}{2}$. The dashed curve is a Gaussian fit to all the data.

Generic models of cluster production may be classed into two types. The first, the class of "fragmentation" scenarios in which a system is broken up by a series of binary splittings or some other physical process, has for its order parameter the number or multiplicity of clusters. For such models, it is the multiplicity which exhibits different $\Delta$-scaling regimes if the system has different phases. The second class of models, in which clusters are built up by "aggregation" of smaller constituents, has for its order parameter the size of the largest cluster. As INDRA does not measure the mass but only the atomic number of fragments, we will assume in the following that the largest fragment of each event corresponds to $Z_{\max }$, the fragment with the largest atomic number.

Figure 5 shows the data for the total multiplicity of charged products with $Z \geq 1, M_{t o t}$, for central collisions of $\mathrm{Xe}+\mathrm{Sn}$ from 25 to $100 A \mathrm{MeV}$. In Figure 5 a we plot the natural logarithm of the variance of the measured $M_{t o t}$ distributions as a function of the natural logarithm of the square of the mean value. The estimated statistical errors of these quantities are smaller than the symbols used. If fluctuations obey the universal scaling law Eq. 2, then the data must fall on a straight line of slope $\Delta$ in this plot. It can be seen in Figure 5a that this is true to a fair approximation, and that the variance of the multiplicity 
distribution grows with increasing bombarding energy as $<M_{\text {tot }}>$.

Figure 5b shows that the multiplicity distributions for different bombarding energies collapse to a unique distribution (scaling function) when expressed in terms of the second scaling law (Equation 1 with $\Delta=\frac{1}{2}$ ), as suggested by Figure 5a. It should be noted that examination of an observable's scaling properties in this way is far more constraining than that of Fig. 5a, when the statistics of the data samples allow it. The observed scaling function is very well approximated by a Gaussian distribution (dashed curve on the figure). The scaling properties of total multiplicity fluctuations are therefore the same for all bombarding energies in the range $25-100 A \mathrm{MeV}$. This is true not only for the $\mathrm{Xe}+\mathrm{Sn}$ data but also for all the data we have studied in this paper: see for example the data for ${ }^{58} \mathrm{Ni}+{ }^{58} \mathrm{Ni}$ collisions in Figure 7 a. Therefore the total multiplicity of charged particles for central collisions in this energy range does not show any evidence of 'anomalous' or 'critical' behaviour.
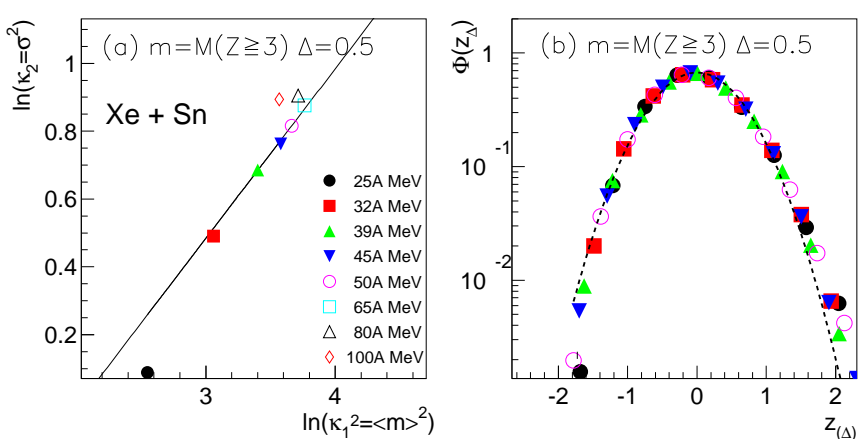

Figure 6: (Color online) As Figure 5 but now for the multiplicity of fragments with $Z \geq 3, M_{Z \geq 3}$. (b) Data for bombarding energies 32-65A MeV expressed in the variables of the second scaling law, i.e. Equation 11 with $\Delta=\frac{1}{2}$.

The multiplicity $M_{t o t}$ is dominated by the multiplicity of light charged particles, $M_{L C P}$, which is typically 3-4 times greater than the multiplicity of IMF $(Z \geq 3)$. However, similar conclusions as for $M_{t o t}$ can be drawn for the IMF multiplicity, $M_{Z \geq 3}$, for which the $\mathrm{Xe}+\mathrm{Sn}$ data are presented in Figure 6, the $\mathrm{Ni}+\mathrm{Ni}$ data in Figure $7 \mathrm{~b}$. Figure 6 a shows that the widths of $M_{Z \geq 3}$ distributions for $\mathrm{Xe}+\mathrm{Sn}$ at bombarding energies 32 to $65 \mathrm{~A} \mathrm{MeV}$ increase with the mean multiplicity according to a $\Delta=\frac{1}{2}$ scaling law, and this is confirmed by Figure $6 \mathrm{~b}$. The width for the $25 \mathrm{~A} \mathrm{MeV}$ system falls below this "systematic" trend, which may indicate that this energy is close to the threshold for multifragmentation in central collisions (the mean multiplicity for this system is approximately 3 fragments with $Z \geq 3$ ), leading to reduced fluctuations of the fragment multiplicity. Recent data obtained with INDRA for the same system at bombarding energies from 8 to $20 \mathrm{~A}$ $\mathrm{MeV}$ will allow to study this point in more detail.

Nevertheless the multiplicity of fragments does show some slightly more interesting features than $M_{t o t}$ : let us remark the "back-bending" in Figure 6a for bombarding energies $>65 \mathrm{AMeV}$, indicating the decrease of mean fragment multiplicity at the highest bombarding energies. The same behaviour is seen more clearly in Figure 7b for $\mathrm{Ni}+\mathrm{Ni}$ collisions above $52 \mathrm{~A} \mathrm{MeV}$. Let us note in passing that the available energy for the maximum of fragment production in the $\mathrm{Ni}+\mathrm{Ni}$ system is here much lower $(13 A \mathrm{MeV})$ than that reported in 60 (17.5A MeV).
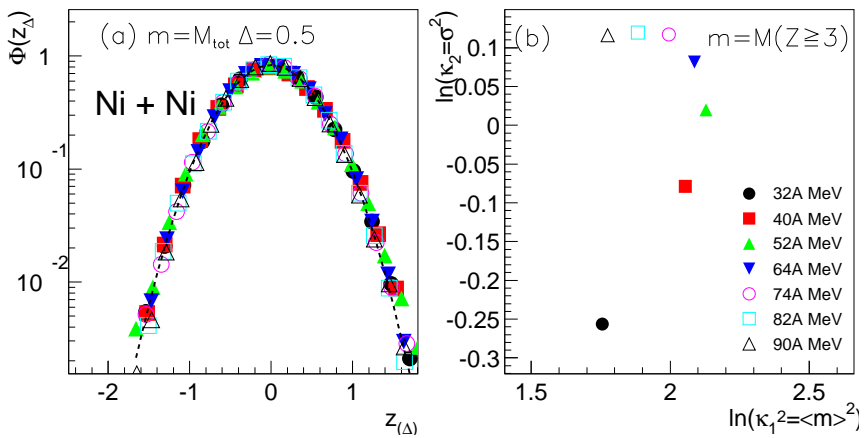

Figure 7: (Color online) Data for well-measured central collisions of $\mathrm{Ni}+\mathrm{Ni}\left(b<0.1 b_{\max }, Z_{\text {det }}>0.8\left(Z_{\text {proj }}+Z_{\text {targ }}\right)\right)$. (a) Distributions of $M_{\text {tot }}$ expressed in the variables of the second scaling law, i.e. Equation 1 with $\Delta=\frac{1}{2}$. The dashed curve refers to a global Gaussian fit to the data (dashed curve). (b) Log-log plot of the variance versus the squared mean value of the distribution of fragment multiplicity, $M_{Z \geq 3}$, for each bombarding energy.

This type of "rise and fall" behaviour has been observed in many different data sets [3, 4, 5, 60]. The absolute value of the maximum mean multiplicity and the energy at which it occurs in central collisions are not only system-dependent [60] but also selection-dependent 15 and detector-dependent. It should be noted that in the framework of the universal fluctuations theory this rise and fall is not consistent with the fragment multiplicity being an order parameter. Rather, in the energy domain for which fluctuations of $M_{Z \geq 3}$ show a regular scaling behaviour (32-65A MeV for $\mathrm{Xe}+\mathrm{Sn})$, it can be seen that this scaling is always of the second kind $\left(\Delta=\frac{1}{2}\right)$ and in this domain the multiplicity distributions all collapse to a unique, quasi-Gaussian scaling function (Figure $6 \mathrm{~b}$ ).

Therefore, neither the total charged multiplicity nor the fragment multiplicity have fluctuations which indicate the presence of different "phases" for central collisions in this energy range. It should be noted, however, that we cannot exclude the possibility of a different behaviour of observables to which we do not have access in this data, for example the true total multiplicity including neutrons. Neither does our result exclude the possibility that in other fragmentation reactions, e.g. at higher bombarding energies and/or in spectator decays rather than central collisions, the total or fragment multiplicity may be the pertinent order parameter. For our data on central collisions between 25 and $100 \mathrm{~A} \mathrm{MeV} \mathrm{how-}$ ever we will from now on only consider the other possible order parameter for fragmentation, the size of the largest fragment. 

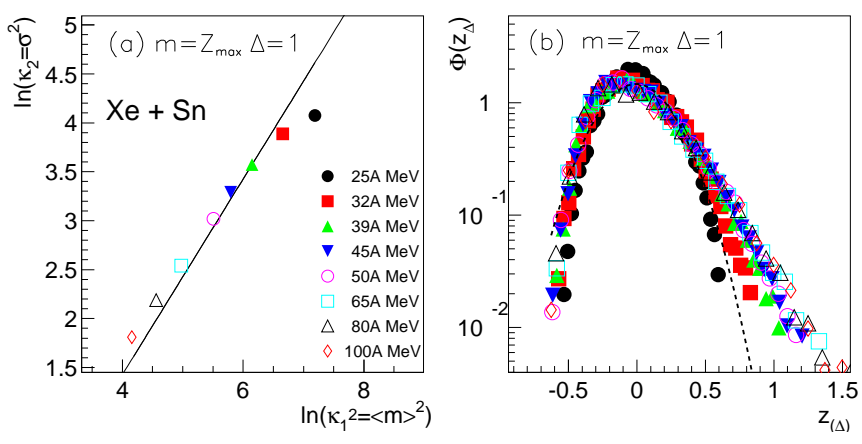

Figure 8: (Color online) As Figure $\mathrm{A}$ (well-measured central $\mathrm{Xe}+\mathrm{Sn}$ collisions) but for the charge $Z_{\max }$ of the heaviest fragment detected event-by-event, and $\Delta=1$.

Looking at the log-log plot of the first two cumulant moments of $Z_{\max }$ (Figure $8 \mathrm{a}$ ) we can suspect some evolution of the scaling behaviour of this observable's fluctuations with increasing beam energy: the data do not appear to fall on a single straight line, but rather seem to be grouped into two "branches" with different slopes. Although most of the data in Figure 8a lie close to a line of slope $\Delta=1$, this does not correspond to a universal scaling law, as all data in Figure $8 \mathrm{~b}$ do not collapse onto a single universal curve under the first scaling law (Equation 1 with $\Delta=1$ ). This confirms that there is a change in the scaling behaviour of $Z_{\max }$ fluctuations with increasing energy. The charge of the largest fragment in each event, and not the fragment multiplicity, seems therefore to be a good candidate for the order parameter of multifragmentation in central collisions at these energies.

\section{Evolution of the scaling behaviour of order parameter fluctuations with incident energy}
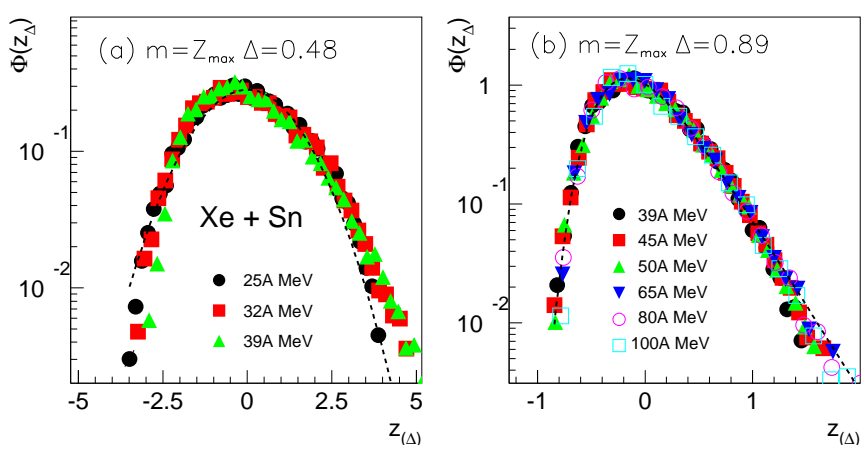

Figure 9: (Color online) (a) $Z_{\max }$ distributions for $\mathrm{Xe}+\mathrm{Sn}$ collisions at $25-39 \mathrm{~A} \mathrm{MeV}$, scaled according to Equation 1 with $\Delta$ given by a linear fit to the data of Figure $8 \mathrm{a}$ of the form $\sigma \sim<m>^{\Delta}$. The dashed curve is a best fit to scaled data using a Gaussian distribution. (b) As (a) but for bombarding energies 39-100A MeV. The dashed curve is a best fit to scaled data using the Gumbel distribution, Eq.5.
Figure 8b shows that the scaled $Z_{\max }$ distributions for $\mathrm{Xe}+\mathrm{Sn}$ collisions from $45 \mathrm{~A} \mathrm{MeV}$ upwards are nearly identical even in the large- $Z_{\max }$ tails which are 2 orders of magnitude less probable than the most probable value of $Z_{\max }$. The $39 \mathrm{~A} \mathrm{MeV}$ data can also be included in this group if the small differences in the tail of the distribution are neglected. However, the $32 \mathrm{~A} \mathrm{MeV}$ distribution is clearly significantly narrower, while the distribution for $25 \mathrm{~A} \mathrm{MeV}$ is evidently of a different form, as can be seen both in the tails and around the maximum (see comments on the comparison of experimental distributions with this technique in Sec.II).

Using the values of $\Delta$ extracted from Fig. 8 a using a linear fit to the two 'branches' (25-39A MeV and 39$100 \mathrm{~A} \mathrm{MeV}$ ) we find a good scaling of distributions using $\sigma \sim\left\langle Z_{\max }\right\rangle^{1 / 2}$ at the lowest beam energies (Figure 9 a), whereas for the higher energies the fluctuations of the size of the largest fragment increase like $\sigma \sim<Z_{\max }>$ (Figure 9b). An approximately equally good scaling for $39 \mathrm{~A} \mathrm{MeV}$ data is achieved in both cases, and this energy may be considered as close to a transition between the two regimes. Figure 9 also shows that the shape of the scaling function changes with increasing beam energy: it is nearly Gaussian at low energy (the dashed curved in Figure 9 a represents a best fit to all data with a Gaussian distribution), but at higher energies it is rather asymmetric with a near-exponential tail for $Z_{\max }$ greater than its most probable value (see Sec. IVE).

These observations establish the size (or charge) of the largest fragment as the most likely "order parameter" for fragment production in central collisions in this energy range. They also show that data can be assigned to one of two regimes depending on the $\Delta$-scaling properties of this order parameter. At low energies systems obey the second-scaling law associated with an ordered phase, while at higher energies the first-scaling law is observed, typical of a disordered phase (large fluctuations) 42. It should be noted that these regimes are defined, not by the average size of the largest fragment produced for a given incident energy (which would be a typical way to define e.g. liquid and gas phases), but rather by the way in which the fluctuations of this quantity evolve compared to its mean value. The identification of the largest fragment size as order parameter also indicates some similarity between fragment production in central collisions and the aggregation models with the second-order phase transition mentioned in Sec. II, whereas the monotonous behaviour of total and fragment multiplicities excludes "shattering" fragmentation models such as FIB [46.

Another, not necessarily incompatible, interpretation of the different $\Delta$ regimes is suggested by Fig. 4. As we remarked in Sec. III B this figure suggests that at beam energies $\lesssim 39 \mathrm{~A} \mathrm{MeV}$ the heaviest fragment in central collisions is an evaporation residue of an (incomplete) fusion reaction. The data presented in Fig. 8 a for incident energies $25-50 A \mathrm{MeV}$ correspond to the points highlighted by a circle in Fig. 4 . Therefore the transition from a $\Delta \sim \frac{1}{2}$ to a $\Delta \sim 1$ branch can be interpreted as being linked 

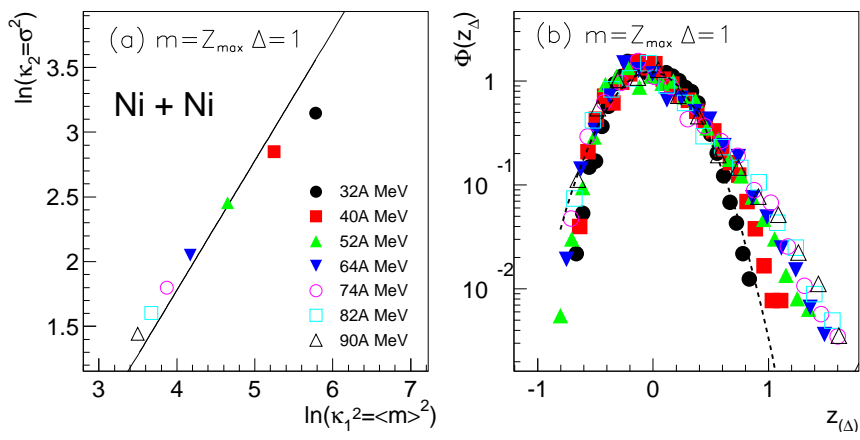

Figure 10: (Color online) As Figure 8 but for collisions of $\mathrm{Ni}+\mathrm{Ni}$ from 32 to $90 A \mathrm{MeV}$ bombarding energy.

to the disappearance of fusion-evaporation residues, and signalling either the onset of fusion-multifragmentation (phase transition) or the onset of transparency in central collisions (incomplete stopping).

It should be noted that the transition energy of around $39 A \mathrm{MeV}$ for the $\mathrm{Xe}+\mathrm{Sn}$ system is slightly higher than that found in 44 (32A MeV). This is due to the different method of selection of 'central collisions'. In this paper we define central collisions based on the amount of energy deposited into the light charged particles' degrees of freedom $\left(E_{t 12}\right)$. As we are dealing with finite systems this reduces the energy available for the fragment degrees of freedom, due to energy conservation. This is consistent with the fact that when central collisions are selected based on fragment degrees of freedom (the fragment kinetic energy flow angle selection of [28, 34, 61]) the multifragmentation regime is observed at lower incident energy for the same system $(32 \mathrm{~A} \mathrm{MeV} \mathrm{for} \mathrm{Xe}+\mathrm{Sn}$ in [35]), as is the transition to the $\Delta=1$ scaling regime.

The observed scaling properties of $Z_{\max }$ fluctuations are confirmed by the $\mathrm{Ni}+\mathrm{Ni}$ data, as shown by Figure 10 and Figure 11. Most data follow a $\Delta \approx 1$ scaling law for $Z_{\max }$ fluctuations, except at the lowest energies. The data do not all collapse to a single scaling function in terms of a $\Delta=1$ law (Figure $10 \mathrm{~b}$ ), but only for bombarding energies $E \geq 52 A \mathrm{MeV}$ (Figure 11b). The similarity between the scaling functions observed for the two different systems should be noted, both in the "ordered" $\left(\Delta \sim \frac{1}{2}\right)$ and "disordered" $(\Delta \sim 1)$ regimes (Figures 9 \& 11 and Table $\mathbb{1}$.

\section{System-size dependence of energy of transition from "ordered" to "disordered" regime}

We observe for the $\mathrm{Xe}+\mathrm{Sn}$ and $\mathrm{Ni}+\mathrm{Ni}$ data that the energy ranges corresponding to the different regimes are not the same for two systems of different total mass, the "transition" occurring around $39 \mathrm{~A} \mathrm{MeV}$ for Xe+Sn and $52 A \mathrm{MeV}$ for $\mathrm{Ni}+\mathrm{Ni}$. The "disordered" regime begins at lower incident (or available) energy for the heavier system. This tendency is confirmed by the data for $\mathrm{Ar}+\mathrm{KCl}$ and $\mathrm{Au}+\mathrm{Au}$ (figures 12 and 13). The former has a total
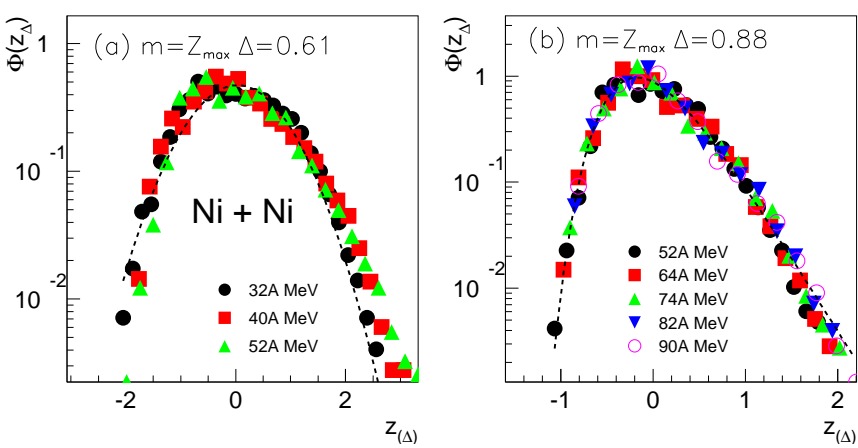

Figure 11: (Color online) (a) $Z_{\max }$ distributions for $\mathrm{Ni}+\mathrm{Ni}$ collisions at $32-52 \mathrm{~A} \mathrm{MeV}$, scaled according to Equation 1 with $\Delta$ given by a linear fit of the form $\sigma \sim<m>^{\Delta}$. The dashed line is a best fit using a Gaussian distribution. (b) As (a) but for energies $52-90 \mathrm{~A} \mathrm{MeV}$. The dashed line is a best fit using the Gumbel distribution, Eq.5.
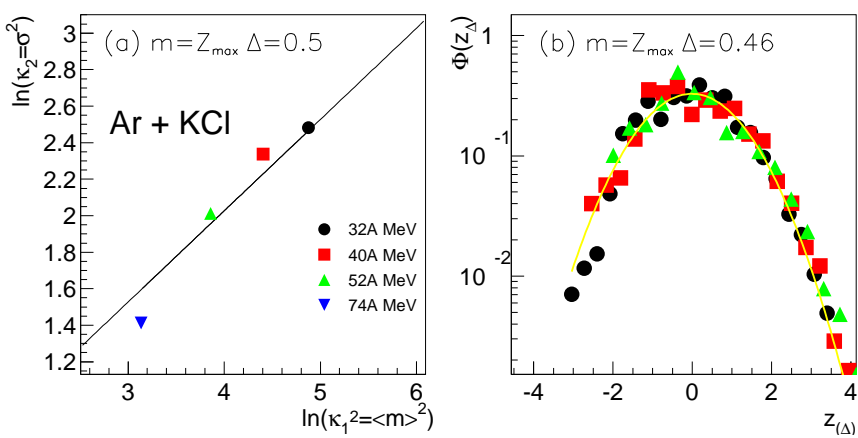

Figure 12: (Color online) As Figure 8 but for collisions of $\mathrm{Ar}+\mathrm{KCl}$ from 32 to $74 A \mathrm{MeV}$ bombarding energy.

mass smaller than both $\mathrm{Xe}+\mathrm{Sn}$ and $\mathrm{Ni}+\mathrm{Ni}$. The largest fragments produced in central collisions of ${ }^{36} \mathrm{Ar}+\mathrm{KCl}$ follow quite closely a $\Delta \sim \frac{1}{2}$ scaling behaviour for beam energies up to at least $52 \mathrm{~A} \mathrm{MeV}$ (Figure 12a), while the data for $74 A \mathrm{MeV}$, which deviate from the overall trend, may indicate a transition to the $\Delta \sim 1$ regime occurs somewhere between these last two available data points. The data for $32-52 \mathrm{~A} \mathrm{MeV}$ exhibit very similar near-Gaussian probability distributions. Although we can not have much confidence in a scaling law established for only two data points let us remark in passing that the scaling behaviour observed for data at 52 and $74 A \mathrm{MeV}$ is approximately a $\Delta \sim 1$ scaling with a scaling function of a form similar to that observed for the other "disordered" regime data, indicating that the transition energy for this system is probably somewhere between 52 and $74 A \mathrm{MeV}$, higher than for the heavier systems.

On the other hand, for the much heavier ${ }^{197} \mathrm{Au}+{ }^{197} \mathrm{Au}$ system a $\Delta=1$ scaling law is observed for fluctuations of the size of the largest fragment in each event for all studied beam energies (Figure 13). Even at the lowest energy $(40 \mathrm{~A} \mathrm{MeV})$ the scaling of the entire $Z_{\max }$ distribution with the other energies is excellent. For this system it may be that the beam energy of the transition 
from the $\Delta=\frac{1}{2}$ regime, if it exists, to $\Delta=1$ occurs at a lower energy than the lowest energy available for study in our data, which may explain why we do not observe an "ordered" regime for this system. Of course it is equally possible that no such regime exists for this system. Indeed, following the interpretation of Sec IV of the disappearance of fusion-evaporation residues, we would not expect fusion to occur for a system as heavy as $\mathrm{Au}+\mathrm{Au}$ at any incident energy.
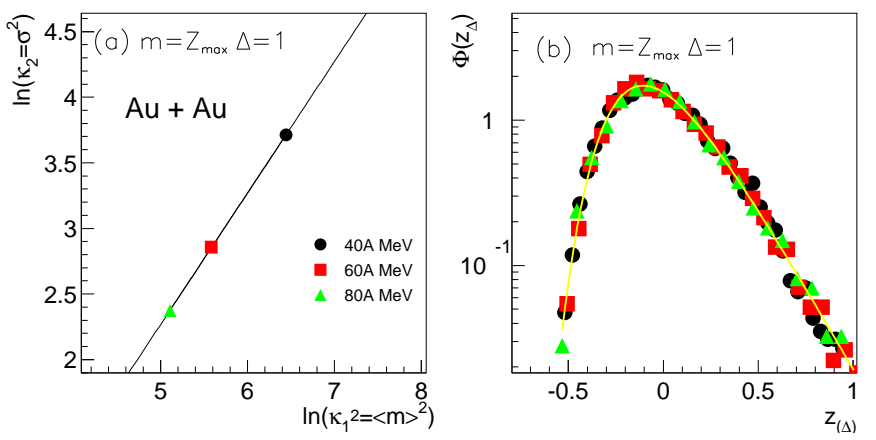

Figure 13: (Color online) As Figure 8 but for collisions of $\mathrm{Au}+\mathrm{Au}$ from 40 to $80 \mathrm{~A} \mathrm{MeV}$ bombarding energy.

We therefore observe that the bombarding energy (or available energy) at which there is a transition from the "ordered" to the "disordered" regime decreases with increasing total mass of the system under study. This is represented in Figure 14. If one interprets our results in

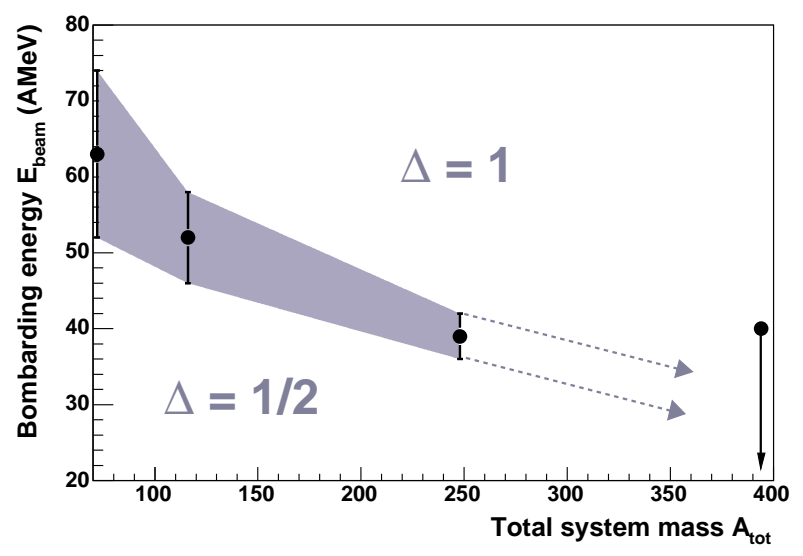

Figure 14: Dependence on bombarding energy and total system mass of the frontier between the two $\Delta$-scaling regimes observed in this work for very central collisions.

the framework of the universal fluctuations theory, i.e. in terms of a 2nd order phase transition, one would expect the energy at which one phase is replaced by the other to be related to the critical temperature of the corresponding system. The definition of this quantity for finite, charged, systems such as nuclei has received much theoretical attention. On the one hand, in the absence of Coulomb forces, a pseudo-critical temperature (corresponding to a large peak in the finite system specific heat) may be defined which is smaller than the infinite matter value $T_{C}$, and increases as the size of the system approaches the infinite matter limit 62. On the other hand, calculations including the Coulomb repulsion show that the maximum temperature that an equilibrated hot nucleus can support, $T_{\text {lim }}$, decreases for heavier nuclei, due to their increasing nuclear charge [63]. A recent systematic study of a wide range of data on so-called caloric curves by Natowitz et al 64 has shown that the temperature and excitation energy at which a plateau is observed in these curves decreases with increasing mass of the primary excited nucleus, and that such behaviour is consistent with theoretical predictions for the limiting temperatures, $T_{\text {lim }}$.

In the present analysis we chose not to study this question in more detail, as to do so would require: (i) to show that the fragment production is thermally-driven; (ii) to identify the thermal (sub-)system in each case; and (iii) to deduce the mass, charge, excitation energy and temperature of this (sub-)system. Each step would require us to make important hypotheses about the formation and decay properties of fragments in the collisions under study, taking us far from our initial goal of determining as much information as possible on the nature of fragment production with a minimum number of suppositions. Moreover, the observed effect, which depends on the entrance channel total mass and available/bombarding energy may have a completely different origin. Therefore we will limit ourselves to the observation that the transition from "ordered" to "disordered" regime takes place at a lower available energy for systems of greater total mass.

\section{E. Detailed study of the form of the scaling functions}

The results of the analysis for all the systems presented here are summarized in Table If. Systems have been grouped according to their observed $\Delta$-scaling behaviour, $\Delta \sim \frac{1}{2}$ at the lowest beam energies and $\Delta \sim 1$ at the highest. In order to get some quantitative information on the form of the scaling function in each case, we calculated the overall coefficient of skewness, $\gamma$, and the kurtosis, $\kappa$, for each system in each regime, using the following definitions 65

$$
\begin{gathered}
\gamma=\frac{<z_{(\Delta)}^{3}>}{\sigma^{3}} \\
\kappa=\frac{<z_{(\Delta)}^{4}>}{\sigma^{4}}-3
\end{gathered}
$$

Larger skewness values indicate more asymmetric distributions, while the kurtosis measures the deviation of the distribution from the Gaussian form $(\kappa=0$ being a perfect Gaussian). The values shown in the table confirm 
Table I: Fluctuation scaling exponent $\Delta$, coefficient of skewness $\gamma$, kurtosis $\kappa, \chi^{2}$ for global fits to data with Gaussian $\left(\Delta \sim \frac{1}{2}\right)$ and Gumbel $(\Delta \sim 1)$ distributions, and scaling function tail exponent $\hat{\nu}\left(\chi^{2}\right)$ (see Section II), for the scaling functions shown in Figures 1, 11, 12 and 13 .

\begin{tabular}{lc|rrrrr}
\hline \hline System & $E_{\text {inc }}(\mathrm{AMeV})$ & $\Delta$ & $\gamma$ & $\kappa$ & $\chi^{2}$ & $\hat{\nu}\left(\chi^{2}\right)$ \\
\hline $\mathrm{Xe}+\mathrm{Sn}$ & $25-39$ & 0.48 & 0.45 & 0.66 & 27 & - \\
$\mathrm{Ni}+\mathrm{Ni}$ & $32-52$ & 0.61 & 0.48 & 0.64 & 72 & - \\
$\mathrm{Ar}+\mathrm{KCl}$ & $32-52+$ & 0.46 & 0.33 & 0.64 & 63 & - \\
\hline $\mathrm{Au}+\mathrm{Au}$ & $40-80$ & 1.00 & 0.83 & 1.39 & 1.8 & $0.92 \pm .09(1.3)$ \\
$\mathrm{Xe}+\mathrm{Sn}$ & $39-100$ & 0.89 & 0.85 & 1.20 & 11 & $1.20 \pm .03(1.2)$ \\
$\mathrm{Ni}+\mathrm{Ni}$ & $52-90$ & 0.88 & 0.84 & 1.30 & 45 & $1.54 \pm .04(7)$ \\
\hline \hline
\end{tabular}

our observation that the higher-energy, $\Delta \sim 1$-scaling data present more asymmetric, less Gaussian probability distributions than those at lower energy. It can also be seen that, quantitatively, the scaling functions corresponding to the two regimes for systems of different masses have very similar forms, confirming the fact that data for different systems do indeed collapse on to a single distribution.

In the "ordered" regime the fluctuations of the size of the largest fragment show a significant deviation from a Gaussian distribution, on the contrary to the near-perfect Gaussian distributions that we observe for total and fragment multiplicities (Figures 5, 6 and 7). For the "disordered" regime the deviation from the Gaussian form is large and the shape of the distribution function is well reproduced by a Gumbel distribution,

$$
\Phi\left(z_{\Delta}\right) \sim \exp -\left(z-\mathrm{e}^{-z}\right)
$$

This is shown by the dashed curves in Figures 9 $\mathrm{b}$ and $11 \mathrm{~b}$, and the grey curve in Figure $13 \mathrm{~b}$, which represent the best fits to these data using Equation 5. The overall agreement can be seen to be quite good, and excellent for the $\mathrm{Au}+\mathrm{Au}$ data where $\chi^{2}=1.8$ is achieved. Some significant deviations can be seen however in the tail of the distributions for the lighter systems $\mathrm{Ni}+\mathrm{Ni}$ and $\mathrm{Xe}+\mathrm{Sn}$ : the data seem to have a faster-than-exponential fall-off for large (positive) fluctuations about the mean value.

To confirm this in a quantitative manner we performed fits to the tails of these functions with the asymptotic scaling function form $\exp -z^{\hat{\nu}}$ (see Table Ii). They show that the exponent $\hat{\nu}$ is very close to 1 for the $\mathrm{Au}+\mathrm{Au}$ data, confirming the observation of an exponential tail compatible with the Gumbel distribution, while for $\mathrm{Ni}+\mathrm{Ni}$ and $\mathrm{Xe}+\mathrm{Sn}$ data we find $1<\hat{\nu}<2$. Let us remark in passing that, just as $\Delta$ seems to increase toward the asymptotic value of 1 with increasing system mass in the "disordered" regime, it is possible that $\hat{\nu}$ also has a systematic mass dependence, and decreases towards an asymptotic value of 1 (exponential tail) for the heaviest system.

We have clearly and quantitatively established the form of the scaling functions in the two regimes, and in the data presented here we do not observe any deviation from these "canonical" forms, quasi-Gaussian at low energy and quasi-Gumbel at high energy. We do not, therefore, have any information on the nature of the transition between the two regimes: as we discussed in Section III we cannot be certain of the form of the scaling function at the critical point, but one may see a sharp decrease of the large- $z$ tails (faster than $\exp -z^{2}$ ), or an order parameter distribution (OPD) with an exponential large- $z$ tail but significantly different from the Gumbel distribution around the maximum and below [66]. On the other hand, for a first-order phase transition with a passage through the coexistence region, although the scaling behaviour of the OPD is not well established [42, 67], by definition in this case the order parameter should present a bimodal (double-humped or very wide) distribution. More detailed study of existing and new data around the transition energy of $39 A \mathrm{MeV}$ (for the $\mathrm{Xe}+\mathrm{Sn}$ system) may yet reveal such features.

\section{F. Dependence of the observed scaling behaviour on the violence/centrality of collisions}
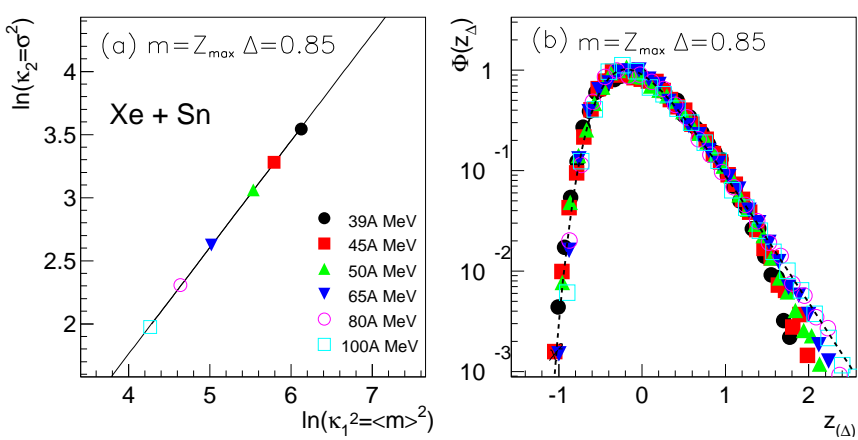

Figure 15: (Color online) As Figure 8, but for collisions with an estimated centrality of $b<0.2 b_{\max }$.

The results presented above come from a wide-ranging set of data concerning different system energies and masses, but they represent only $1 \%$ of the total measured cross-section due to our centrality cut. The representativity of the observed scaling behaviour is far from 
certain in this case, as it is well known that data selected by cuts in distribution tails are prone to serious autocorrelation effects due to conservation laws 15, 68. We therefore have to study the dependence of our findings on the strictness of our centrality cuts. In Figure 15 the data for the same Xe+Sn collisions as in Figure $9 \mathrm{~b}$ have been analyzed with a slightly relaxed centrality cut, $b<0.2 b_{\max }$. The effect on the apparent value of $\Delta$ can be seen from the cumulant moments plot, Figure 15a: relaxing the centrality condition gives an apparent scaling law with a smaller value of $\Delta$, which is here $\Delta \approx 0.85$ instead of $\Delta=0.89$ (see Table il). A similar dependence on the estimated centrality of collisions was observed in 44, and interpreted in terms of a smaller excitation energy of fragmenting systems in less central collisions. However, as Figure 15b shows, the $\Delta$-scaling actually begins to break down for these less central data, as can be seen in the high- $z_{(\Delta)}$ tail of the "scaling" function.
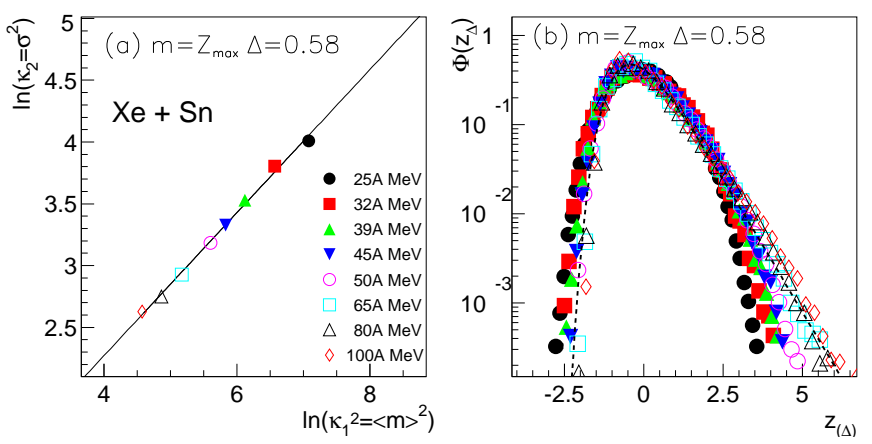

Figure 16: (Color online) As Figure 15, but for collisions with an estimated centrality of $b<0.4 b_{\max }$.

If the centrality condition is relaxed even further, $b<0.4 b_{\max }$ (Figure 16), we first observe that now apparently all of the data for $\mathrm{Xe}+\mathrm{Sn}$ is compatible with an approximate second-scaling law $(\Delta=0.58)$, without any change of scaling regime between 25 and $100 \mathrm{~A} \mathrm{MeV}$. However we can not assign the data to a single "ordered" regime because in fact this is not true $\Delta$-scaling: the different distributions no longer collapse to a universal curve (Figure 16b).

Therefore we observe universal fluctuations and behaviour of the $Z_{\max }$ observable compatible with it being an order parameter for nuclear multifragmentation only for very central collisions of symmetric systems for which one may suppose a near-to-total overlap of the projectile and target in the entrance channel. We have not, up to now, observed an equivalent scaling for quasiprojectile residues in mid-peripheral to peripheral collisions. This may be because in such reactions the fragment production is far more sensitive to entrance channel effects, which vary greatly as a function of the colliding nuclei and their energies. If so, a meaningful comparison between different systems is harder to achieve, at least with our rather "global" approach. In head-on collisions, on the other hand, the fragmentation of the projectile and target may be virtually a statistical (although not necessarily thermal) process, far less sensitive to the details of the reaction, and therefore more amenable to reveal features which are independent of the system under study.

\section{SUMMARY}

We have studied nuclear multifragmentation data obtained with the INDRA $4 \pi$ array for collisions of symmetric systems of total mass $A_{t o t} \sim 75-400$ at bombarding energies from 25 to $100 \mathrm{~A} \mathrm{MeV}$. Using the total transverse energy of light charged particles, $E_{t 12}$, as a measure of collision violence, we deduced the evolution of these reactions with beam energy, impact parameter and system size from the experimentally measured correlations between the charge of the largest fragment detected in each event, $Z_{\max }$, and $E_{t 12}$. For all data presented in this work these correlations are dominated by reactions leading to a projectile-like fragment whose size decreases with increasing collision violence. In the case of the heaviest projectiles (Xe, Au) fission of the moderately excited quasi-projectile modify this picture. For higher excitation energies the opening of the quasi-projectile multifragmentation channel may exhibit a bimodal behaviour which has been evidenced [69 in selecting complete detection events. On the other hand, in central collisions for a few percent of the measured cross-section "heavy" residues are produced in the systems $\mathrm{Ar}+\mathrm{KCl}, \mathrm{Ni}+\mathrm{Ni}$ and $\mathrm{Xe}+\mathrm{Sn}$, suggesting incomplete fusion of projectile and target, for beam energies which are not too high $(\leq 52 A \mathrm{MeV}$ for $\mathrm{Ar}+\mathrm{KCl}, \leq 40 A \mathrm{MeV}$ for $\mathrm{Ni}+\mathrm{Ni}, \leq 32 A$ $\mathrm{MeV}$ for $\mathrm{Xe}+\mathrm{Sn})$.

Using a model-independent analysis based on the theory of universal fluctuations of the order parameter for finite systems, we tested the most violent collisions for signals that the fragment production may be related to a phase transition. Following the results of 44 we first confirmed that, of the two possible order parameters for a critical fragmentation process, it is the charge (size) of the largest fragment, $Z_{\max }$, and not the total or IMF multiplicities $M_{t o t}$ or $M_{Z>3}$, which has a behaviour of the scaling properties of its fluctuations compatible with its being an order parameter for a critical fragmentation process. Indeed, we have shown that the event-byevent distribution of $Z_{\max }$ allows to sort data into two fluctuation-scaling regimes defined by the value of the scaling exponent $\Delta$, which is approximately equal to $\frac{1}{2}$ at low energies and tends towards the asymptotic value 1 at high energies with increasing total system size. These regimes are equally well characterised by a distinctive form of the scaling function $\Phi\left(z_{(\Delta)}\right)$. At low energies this function, although more symmetric than that seen at higher energy, is significantly different from the Gaussian form. The deviation is quantitatively the same for the three different-sized systems $(\mathrm{Ar}+\mathrm{KCl}, \mathrm{Ni}+\mathrm{Ni}$ and $\mathrm{Xe}+\mathrm{Sn}$ ) for which we observed this low-energy regime. In the high-energy regime the scaling function is more 
asymmetric and tends towards the asymptotic form of the Gumbel distribution with increasing system mass. This evolution concerns mainly the large- $Z_{\max }$ tail of the distribution which falls off more slowly for heavier systems, becoming exponential. For the $\mathrm{Au}+\mathrm{Au}$ system $\Phi\left(z_{(\Delta)}\right)$ is an almost perfect Gumbel distribution.

The bombarding energy at which the passage from one regime to the other is situated decreases as the total system mass (and charge) increases. This is the contrary of the expected behaviour if this transition were related to the critical temperature of the systems under study, which would increase with the mass, but is on the other hand consistent with the observation of decreasing limiting temperatures for finite nuclei [64] due to the increase in Coulomb energy for heavy nuclei. However, we can only speculate whether this is the reason for our observation, as our analysis does not depend on any model of fragment production such as supposing it to be equilibrated or thermally-driven, and therefore it does not give any information on whether these conditions are met or not. On the other hand, data strongly suggest that the dependence on entrance channel mass and bombarding energy of the two regimes is closely linked to the disappearance of heavy residues in central collisions.

Although this analysis allows to establish the existence of two distinct regimes in multifragmentation reactions based on the scaling properties of the fluctuations of the largest fragment, it has not given any information on the passage from one regime to the other. In the data studied so far we observe only order parameter distribu- tions which are compatible with weakly-correlated systems, i.e. far from the critical point. It may be that the general survey of a wide range of data presented here is not sufficiently detailed to reveal such features, or that data taken in small bombarding energy steps around the "transition energy" is necessary to track the evolution of the order parameter distribution. Such additional data for the $\mathrm{Xe}+\mathrm{Sn}$ system have been measured recently and are currently under analysis. It is our feeling that the present work constitutes a solid basis for further study of the question of criticality in nuclear multifragmentation data.

\section{Acknowledgments}

We would like to thank M. Płoszajczak for fruitful discussions and the support and interest he has shown in our work.

We thank also the staff of the GANIL and GSI Accelerator facilities for their support during the experiments. This work was supported by the European Community under contract No. ERBFMGECT950083, and by the Commissariat à l'Energie Atomique, the Centre National de la Recherche Scientifique, the Ministère de l'Education Nationale, the Conseil Régional de Basse Normandie and the Association Française des Femmes Diplômées des Universités.
[1] R. T. de Souza et al., Phys. Lett. B268, 6 (1991).

[2] D. R. Bowman et al., Phys. Rev. Lett. 67, 1527 (1991).

[3] C. Ogilvie et al., Phys. Rev. Lett. 67, 1214 (1991).

[4] M. B. Tsang et al., Phys. Rev. Lett. 71, 1502 (1993).

[5] G. F. Peaslee et al., Phys. Rev. C49, R2271 (1994).

[6] G. Bertsch and P. J. Siemens, Phys. Lett. B126, 9 (1983).

[7] G. Fai, L. P. Csernai, J. Randrup, and H. Stocker, Phys. Lett. B164, 265 (1985).

[8] G. Peilert et al., Phys. Rev. C39, 1402 (1989).

[9] J. E. Finn et al., Phys. Rev. Lett. 49, 1321 (1982).

[10] R. W. Minich, Phys. Lett. B118, 458 (1982).

[11] M. E. Fisher, Physics (N.Y.) 3, 255 (1967).

[12] W. Friedman and W. G. Lynch, Phys. Rev. C28, 16 (1983).

[13] R. J. Charity et al., Nucl. Phys. A483, 371 (1988).

[14] D. Durand et al., Nucl. Phys. A541, 266 (1992).

[15] W. J. Llope et al., Phys. Rev. C51, 1325 (1995).

[16] W. Friedman, Phys. Rev. C42, 667 (1990).

[17] D. H. E. Gross, Rep. Prog. Phys. 53, 605 (1990).

[18] J. P. Bondorf, A. S. Botvina, A. S. Iljinov, I. N. Mishustin, and K. Sneppen, Phys. Rep. 257, 133 (1995).

[19] X. Campi, Phys. Lett. B208, 351 (1988).

[20] A. Mekjian et al., Phys. Rev. C57, 1361 (1998).

[21] J. Richert and P. Wagner, Phys. Rept. 350, 1 (2001).

[22] L. G. Moretto and G. J. Wozniak, Ann. Rev. Nucl. Part. Sci. 43, 379 (1993).

[23] B. Lott et al., Phys. Rev. Lett. 68, 3141 (1992).
[24] J. Toke et al., Phys. Rev. Lett. 75, 2920 (1995).

[25] J. F. Dempsey et al., Phys. Rev. C54, 1710 (1996).

[26] G. J. Kunde et al., Phys. Rev. Lett. 77, 2897 (1996).

[27] Y. Larochelle et al., Phys. Rev. C53, 823 (1996).

[28] N. Marie et al. (INDRA collaboration), Phys. Lett. B391, 15 (1997).

[29] J. Łukasik et al. (INDRA collaboration), Phys. Rev. C55, 1906 (1997).

[30] R. Nebauer, J. Aichelin, et al. (INDRA collaboration), Nucl. Phys. A658, 67 (1999).

[31] E. Plagnol et al. (INDRA collaboration), Phys. Rev. C61, 014606 (1999).

[32] T. Lefort et al. (INDRA collaboration), Nucl. Phys. A662, 397 (2000).

[33] B. Djerroud et al., Phys. Rev. C64, 034603 (2001).

[34] J. D. Frankland et al. (INDRA collaboration), Nucl. Phys. A689, 905 (2001).

[35] J. D. Frankland et al. (INDRA collaboration), Nucl. Phys. A689, 940 (2001).

[36] B. Borderie et al. (INDRA collaboration), Phys. Rev. Lett. 86, 3252 (2001).

[37] N. Bellaize, O. Lopez, J. P. Wieleczko, D. Cussol, G. Auger, C. Bacri, F. Bocage, B. Borderie, R. Bougault, B. Bouriquet, et al. (INDRA collaboration), Nucl. Phys. A 709, 367 (2002).

[38] J. Colin, D. Cussol, J. Normand, N. Bellaize, R. Bougault, A. M. Buta, D. Durand, O. Lopez, L. Man- 
duci, J. Marie, et al. (INDRA collaboration), Phys. Rev. C 67, 064603 (2003).

[39] J. L. Charvet, R. Dayras, D. Durand, O. Lopez, L. Nalpas, A. van Lauwe, C. Volant, D. Cussol, and D. Doré, Nucl. Phys. A730, 431 (2004).

[40] R. Botet, M. Płoszajczak, and V. Latora, Phys. Rev. Lett 78, 4593 (1997).

[41] R. Botet and M. Płoszajczak, J. of Sol-Gel Science and Technology 15, 167 (1999).

[42] R. Botet and M. Płoszajczak, Phys. Rev. E62, 1825 (2000).

[43] R. Botet and M. Płoszajczak, Nucl. Phys. Proc. Suppl. 92, 101 (2001), hep-ph/0009233.

[44] R. Botet et al., Phys. Rev. Lett. 86, 3514 (2001).

[45] E. Gumbel, Statistics of extremes (Columbia University Press, 1958).

[46] R. Botet and M. Płoszajczak, Phys. Rev. Lett. 69, 3696 (1992).

[47] J. Pouthas, B. Borderie, R. Dayras, E. Plagnol, M.-F. Rivet, F. Saint-Laurent, J.-C. Steckmeyer, et al., Nucl. Instr. and Meth. in Phys. Res. A357, 418 (1995).

[48] J. C. Steckmeyer, D. Cussol, J. Duchon, J. Gautier, J. Laville, P. L. Botlan, A. Leconte, J. Lelandais, V. Métivier, P. Mosrin, et al., Nucl. Instr. and Meth. in Phys. Res. A361, 472 (1995).

[49] J. Pouthas, A. Bertaut, B. Borderie, P. Bourgault, B. Cahan, G. Carles, D. Charlet, D. Cussol, R. Dayras, M. Engrand, et al., Nucl. Instr. and Meth. in Phys. Res. A369, 222 (1995).

[50] G. Tăbăcaru, B. Borderie, A. Ouatizerga, M. Parlog, M.F. Rivet, et al. (INDRA collaboration), Nucl. Instr. and Meth. in Phys. Res. A428, 379 (1999).

[51] M. Pârlog, B. Borderie, M.-F. Rivet, G. Tabacaru, A. Chbihi, M. Elouardi, N. Leneindre, O. Lopez, E. Plagnol, L. Tassan-Got, et al. (INDRA collaboration), Nucl. Instr. and Meth. in Phys. Res. A482, 674 (2002).

[52] M. Pârlog, B. Borderie, M.-F. Rivet, G. Tabacaru, A. Chbihi, M. Elouardi, N. Leneindre, O. Lopez,
E. Plagnol, L. Tassan-Got, et al. (INDRA collaboration), Nucl. Instr. and Meth. in Phys. Res. A482, 693 (2002).

[53] A. Trzciński, J. Łukasik, W. F. J. Müller, W. Trautmann, B. Zwiegliński, G. Auger, C. O. Bacri, M. L. Begemann-Blaich, N. Bellaize, R. Bittiger, et al. (INDRA and ALADIN collaborations), Nucl. Inst. Meth. A501, 367 (2003).

[54] V. Métivier et al. (INDRA collaboration), Nucl. Phys. A672, 357 (2000).

[55] F. Bocage et al. (INDRA collaboration), Nucl. Phys. A676, 391 (2000).

[56] A. Ono, S. Hudan, A. Chbihi, and J. D. Frankland, Phys. Rev. C66, 014603 (2002).

[57] A. Le Fevre et al. (INDRA and ALADIN collaborations), Nucl. Phys. A735, 219 (2004).

[58] J. D. Frankland et al. (INDRA collaboration), in Proc. XL Int. Wint. Meet. On Nuclear Physics, edited by I.Iori (Ricerca scientifica ed educazione permanente, 2002), nucl-ex/0202026.

[59] C. Cavata et al., Phys. Rev. C42, 1760 (1990).

[60] D. Sisan et al., Phys. Rev. C63, 027602 (2001).

[61] J. Lecolley et al., Phys. Lett. B391, 317 (1994).

[62] H. Jaqaman, A. Mekjian, and L. Zamick, Phys. Rev. C29, 2067 (1984).

[63] S. Levit and P. Bonche, Nucl. Phys. A437, 426 (1985).

[64] J. B. Natowitz et al., Phys. Rev. C65, 034618 (2002), nucl-ex/0106016.

[65] B. Roe, Probability and statistics in experimental physics (Springer-Verlag New York, Inc., 1992).

[66] R. Botet and M. Płoszajczak (in preparation).

[67] J. M. Carmona, J. Richert, and P. Wagner, Phys. Lett. B531, 71 (2002).

[68] L. Phair et al., Nucl. Phys. A548, 489 (1992).

[69] B. Tamain, M. Pichon, et al. (INDRA collaboration), in Proc. IWM2003 (Int. Work. on Multifragmentation and Related Topics) (Istituto Nazionale di Fisica Nucleare, 2003). 